\title{
Índice de internación ajustado por riesgo en hospitales públicos de Costa Rica
}

\section{Risk-Adjusted Hospitalization Rate in Public Hospitals in Costa Rica}

\section{Índice de internamento ajustado por risco em hospitais públicos na Costa Rica}

Fecha de recepción: 17-03-16 Fecha de aceptación: 12-09-16 Disponible en línea: 30-11-16 doi:10.11144/Javeriana.rgyps15-31.iiar

Cómo citar este artículo:

Morera-Salas M. Índice de internación ajustado por riesgo en hospitales públicos de Costa Rica. Rev.

Gerenc. Polit. Salud. 2016; 15(31): 202-214. http://dx.doi.org/10.11144/Javeriana.rgyps15-31.iiar

Melvin Morera-Salas**

Artículo de investigación. Estudio realizado por la Caja Costarricense de Seguro Social entre enero y mayo del 2016. Doctor en Economía de la Salud, Dirección Compra de Servicios de Salud, Caja Costarricense de Seguro Social Dirección: San José, Costa Rica. Apartado Postal 332-1000, San José. Correo electrónico: mmoreras@ccss.sa.cr correo alternativo: mmoreras@gmail.com 


\section{Resumen}

La estancia prolongada constituye un importante problema de gestión hospitalaria. El objetivo de este estudio es estimar un índice que identifique los hospitales con una gestión inadecuada de las estancias. El índice de adecuación de estancias se calcula dividiendo las estancias observadas entre las estancias esperadas en cada hospital. Estas últimas se estiman con un modelo de regresión que considera las características sociodemográficas y clínicas de los pacientes. Se utiliza la base de egresos hospitalarios del seguro público de salud de Costa Rica del 2014. El $43 \%$ de los hospitales tiene una inadecuada gestión de las estancias, dado que registran mayores días de hospitalización que los esperados, de acuerdo a lo que le correspondía por la casuística. Se concluye que la información clínico-administrativa del sistema público de Costa Rica permite estimar un índice de adecuación de estancias, para identificar los hospitales con una gestión inadecuada de las estancias.

Palabras clave: gestión en salud; hospitalización; tiempo de internación; ajuste de riesgo; alta del paciente; Costa Rica

\section{Abstract}

A prolonged hospitalization constitutes an important hospital management problem. The purpose of this study is to calculate an index which identifies the hospitals with an inadequate hospitalization management. The hospitalization adequacy index is calculated by dividing the observed hospitalizations by the expected hospitalizations in each hospital. The latter are calculated with a regression model which takes into account the socio-demographic and clinical characteristics of the patients. We use the basis of hospitalization discharges of the public healthcare system in Costa Rica for $2014.43 \%$ of the hospitals have an inadequate hospitalization management, as they record more hospitalization days than expected according to the corresponding case load. We conclude that the clinical-management information of the Costa Rican public healthcare allows us to estimate a hospitalization adequacy index as to identify hospitals with an inadequate hospitalization management.

Keywords: health management; hospitalization; hospitalization time; risk adjustment; patient discharge; Costa Rica

\section{Resumo}

A estancia prolongada constitui importante problema de gestão hospitalar. O objetivo deste estudo foi estimar um índice que identifique os hospitais com gestão inadequada das estancias. O índice de adequação de estancias é calculado dividindo as estancias observadas entre as estancias esperadas em cada hospital. Estas últimas foram estimadas com um modelo de regressão que considera as características sociodemográficas e clínicas dos pacientes. Utiliza-se a base de egressos hospitalares do seguro público de saúde de Costa Rica de 2014. O 43\% dos hospitais tem inadequada gestão das estancias, dado que registram mais dias de hospitalização do que os esperados, de acordo com o esperado por casuística. Conclui-se que a informação clínico-administrativa do sistema público de Costa Rica permite estimar um índice de adequação de estancias, para identificar os hospitais com gestão inadequada das estancias.

Palavras-chave: gestão em saúde; hospitalização; tempo de internação; ajuste de risco; alta do paciente; Costa Rica 


\section{Introducción}

En los hospitales la cama es uno de los recursos centrales para la prestación de servicios. $\mathrm{Su}$ adecuada utilización es un elemento central en la gestión hospitalaria y, por lo tanto, el uso que se le dé constituye una medida global de la eficiencia hospitalaria.

Un servicio de hospitalización con estancias prolongadas de pacientes puede considerarse un indicador de falta de eficiencia en el flujo de pacientes. Además de la relación directa entre la prolongación de la estancia y la disponibilidad de camas, aumentan los costos de atención, el riesgo de eventos adversos y la mortalidad (1-2).

La estancia media es uno de los indicadores clásicos en la valoración de la eficiencia en la gestión de los recursos de los centros hospitalarios, de amplia utilización a nivel internacional (3-5), dado que resume el aprovechamiento de la cama y la agilidad de los servicios prestados en los hospitales $(1,6)$.

Es evidente, sin embargo, que la simple utilización de la estancia media bruta de los centros solo tiene sentido cuando se comparan centros de similares características estructurales, que tratan parecida casuística y sobre pacientes con el mismo grado de severidad. Esto hace necesario recurrir a un método de "ajuste por riesgo" de la estancia para determinar cuándo se incurre en estancia prolongada por problemas de la atención (7-8).

El primer paso es conocer la tipología de los pacientes, para lo que resulta imprescindible medir la casuística atendida. Los grupos relacionados con el diagnóstico (GRD) brindan información sobre la tipología de pacientes (el tipo de GRD) que atiende el hospital y su grado de complejidad (8-11). Sin embargo, los GRD no siempre están disponibles, dado su alto costo relativo para sistemas de salud de países en desarrollo. Una alternativa es utilizar un modelo de regresión para estimar la estancia esperada en un centro hospitalario en función de las características de los pacientes y algunas condiciones del hospital (7-8).

El objetivo de este estudio es estimar un índice de adecuación de estancias (IAE), utilizando variables sociodemográficas y clínicas de los pacientes que permitan identificar ineficiencias en la gestión en los hospitales públicos de Costa Rica (CCSS).

\section{Materiales y métodos}

El estudio es de tipo observacional descriptivo. Se utiliza como fuente de información la base de datos de egresos hospitalarios del 2014 del Área de Estadística en Salud de la CCSS. Se calcula el IAE para el grupo de veintitrés hospitales generales.

En la estimación del IAE se aplican los siguientes criterios:

- Se excluyeron de la base de datos los egresos que registren valores perdidos de las variables de edad, sexo, categoría de ingreso, estancias, códigos diagnósticos primarios y secundarios, estado civil, condición de salida.

- Para el servicio de cirugía mayor ambulatoria (CMA) se consideró que el caso tiene cero estancias. Esto para reconocer la diferencia de aquellos casos que calificaban como CMA y sin embargo fueron ingresados en algunos hospitales.

- Se obtuvieron las estancias observadas en cada hospital.

- Se estimaron las estancias esperadas, que se obtienen de la suma de las pro- 
babilidades de estadía de cada episodio individual. La probabilidad individual se calcula mediante un modelo de regresión de binomial negativa truncada en cero, que incorpora como variables explicativas: edad, sexo, vía de ingreso al hospital, estado civil, condición de salida del paciente, grupo de diagnóstico CIE10 consignado en el egreso hospitalario, grupo de comorbilidad representado por el índice de Charlson (12).

- El valor del IAE para cada centro hospitalario se obtuvo dividiendo las estancias observadas entre las estancias esperadas y multiplicando por 100 .

- Se calculó el intervalo de confianza al $99 \%$ (IC 99\%) para determinar si el IAE es estadísticamente diferente a $100 \%$. Para el cálculo se utilizó el método de puntuación de Wilson sin corrección por continuidad (13).

Las variables explicativas utilizadas para estimar el modelo de regresión que permitió obtener las estancias esperadas, se detallan a continuación:

- Edad: seis grupos de edad (menor o igual a 5, 6-17 años, 18-44 años, 45-64 años, 65-79 años y 80 años y más). El grupo de edad de referencia fue el de menor o igual cinco años.

- Sexo: sexo del paciente, consignado en el registro del egreso (categoría de referencia hombre).

- Estado civil: casado o en unión libre versus cualquier otro estado civil (categoría de referencia otro estado civil).

- Vía de entrada al hospital: urgencia o ingreso programado (categoría de referencia un ingreso programado).
- Grupo diagnóstico principal: se utilizan veintiún grupos según las categorías de la lista de la Organización Mundial de la Salud (OMS) (14).

- Grupo de comorbilidad: para calcular el grado de comorbilidad del paciente se utilizó el índice de Charlson, el cual se clasificó en tres grupos de acuerdo a la puntuación obtenida: pacientes con valores de 0 o 1 en el índice fueron asignados al grupo 0 , pacientes con puntuaciones 2 , 3 o 4 en el grupo 1 y pacientes con puntajes de 5 y más en el grupo 2. La categoría de referencia es ninguna comorbilidad.

- Condición de salida del paciente: muerte versus otra condición (categoría de referencia otra condición).

El índice de Charlson (12) es una puntuación de comorbilidad general ampliamente utilizada en la investigación clínica y de gestión. Para el presente estudio se emplea según la metodología actualizada de Quan (12).

Se utilizó un modelo binomial negativo, por ser datos de conteo que presentan una sobredispersión — según los datos de la CCSS, en el 2014 la varianza de las estancias hospitalarias es 1.6 veces superior al promedio-. Este modelo se estimó separadamente para la base de datos total y la base excluyendo casos extremos (se consideró como límite superior la media geométrica más dos desviaciones estándar, lo que significó excluir los casos con más de diecinueve días de estancia). A partir de los regresores se derivaron los pesos de cada una de las variables relacionadas estadísticamente con la estancia de los pacientes, así como el valor del intercepto empleado en el cálculo de la estancia esperada en el 2014 de cada uno de los centros analizados.

Un IAE > 100 indica mayores estancias que las que le corresponde por la casuística aten- 
dida; por tanto, los hospitales con valores significativamente superiores a 100 no son deseables, dado que registran una gestión no adecuada de las estancias. Un IAE $\leq$ se presenta cuando un hospital registra estancias iguales o inferiores a las que le corresponde por la complejidad de los casos atendidos.

La estimación del modelo de regresión binomial negativa se realizó en el programa estadístico Stata 12 utilizando la opción de errores estándar robustos para grupos (clustered robust).

\section{Resultados}

\section{Análisis descriptivo}

Durante el 2014 se produjeron 295672 egresos hospitalarios en los veintitrés hospitales generales de la CCSS, con una estancia media de 4.7 días y una desviación estándar de 7.64 días. Las medidas posición muestran que el $25 \%$ de los casos presentó un día de estancia, la mediana fue de dos días y el percentil 75 fue de cinco días. Además, el 95\% de los casos presentaron diecisiete o menos días de estancia.

El 17\% de los casos son cirugía mayor ambulatoria (que en esta investigación se le asignó una estancia de cero).

Las estancias superiores a diecinueve días (casos extremos) representaron un $4.2 \%$ del total de egresos y $28.4 \%$ del total de días estancia.

La estancia media en los hombres (6.03 días) es $58 \%$ más alta que la registrada en mujeres (3.81 días). En la figura 1 se presenta la estancia media por tipo de hospital, segregado por grupos de edad y sexo. Se registra una estancia media mayor en hospitales nacionales en todos los grupos de edad para los diferentes tipos de hospital, excepto para el grupo de edad de ochenta años y más, donde las estancias son relativamente altas en los tres tipos de hospitales y en ambos sexos.

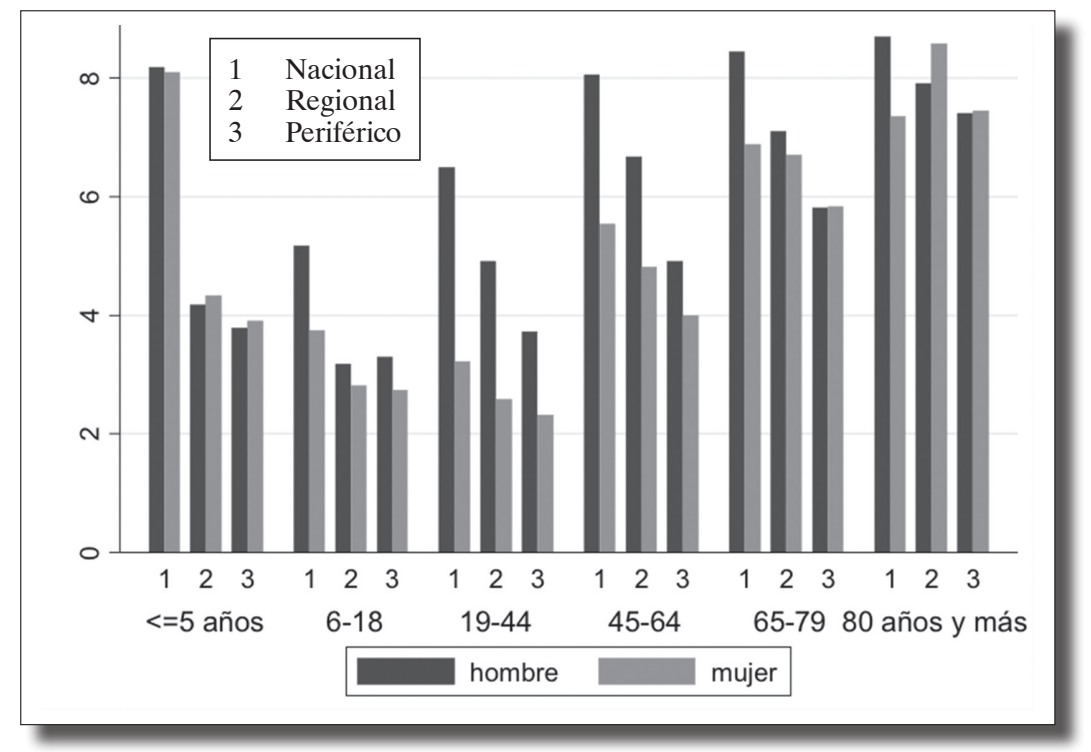

Figura 1. Estancia media POR tipo DE hospital, SEgREgado POR GRUPOS DE EDAD y SEXo, hospitales GenERALES

Fuente: elaboración propia con base en datos del Área de Estadística en Salud, Caja Costarricense de Seguro Social 
Existen diferencias en la estancia entre hombres y mujeres en los grupos de edad intermedios y sobre todo en los hospitales nacionales. La única situación donde la estancia es mayor en mujeres se presenta en hospitales regionales en el grupo de ochenta años y más, y levemente superior en hospitales regionales y periféricos en menores de cinco años.

En la figura 2 se muestra la estancia media por grupo diagnóstico, separado por sexo. Se observa que, exceptuando el grupo de "otros diagnósticos", la estancia media es mayor en los grupos de enfermedades endocrinas, nutricionales y metabólicas y trastornos mentales y del comportamiento.

También se observa en la figura 2 que existe una razón de tasas hombre/mujer muy superior a la unidad en los grupos de enfermedades del sistema nervioso y tumores.

\section{Resultados del modelo}

El análisis de los datos, previo al desarrollo del análisis multivariante, indica la presencia de una sobredispersión. La distribución de estancias presenta una media de 4.7 y una varianza de 7.64, con lo cual el coeficiente de dispersión es claramente superior a 1.

A partir del modelo de regresión binomial negativa, utilizado para estimar el IAE, se determinó que se presenta una mayor estadía en los hombres que en las mujeres, una mayor probabilidad de estancias altas en los grupos de edad avanzada con respecto a los primeros años de vida, y una muy alta probabilidad de permanecer más en el hospital si el paciente ingresa por emergencia, en comparación a si lo hace de forma programada.

Se registró una mayor estancia en los pacientes que presentan un índice de Charlson positivo con respecto al que no presenta comorbilidad. Estar casado o en unión libre es una condición que implica una menor estancia.

Por su parte, los pacientes que fallecen en el hospital tienen una mayor posibilidad de tener una estancia mayor que en cualquier otra condición de salida.

Los resultados no varían sustancialmente, excepto la comorbilidad media, que no resulta significativa, y el grupo de enfermedad

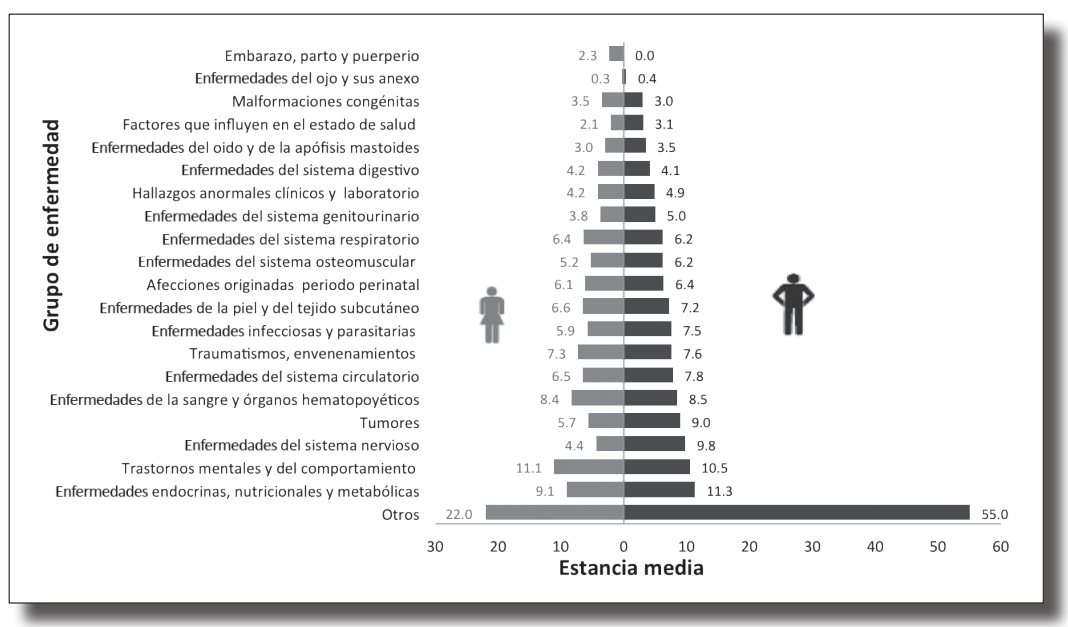

Figura 2. Estancia media por grupo diagnóstico SEParado POR SEXo, Costa Rica, 2014

Fuente: elaboración propia con base en datos del Área de Estadística en Salud, Caja Costarricense de Seguro Social 
Tabla 1. Medidas de asociación entre la estancia hospitalaria y las variables del paciente en Costa Rica, 2014

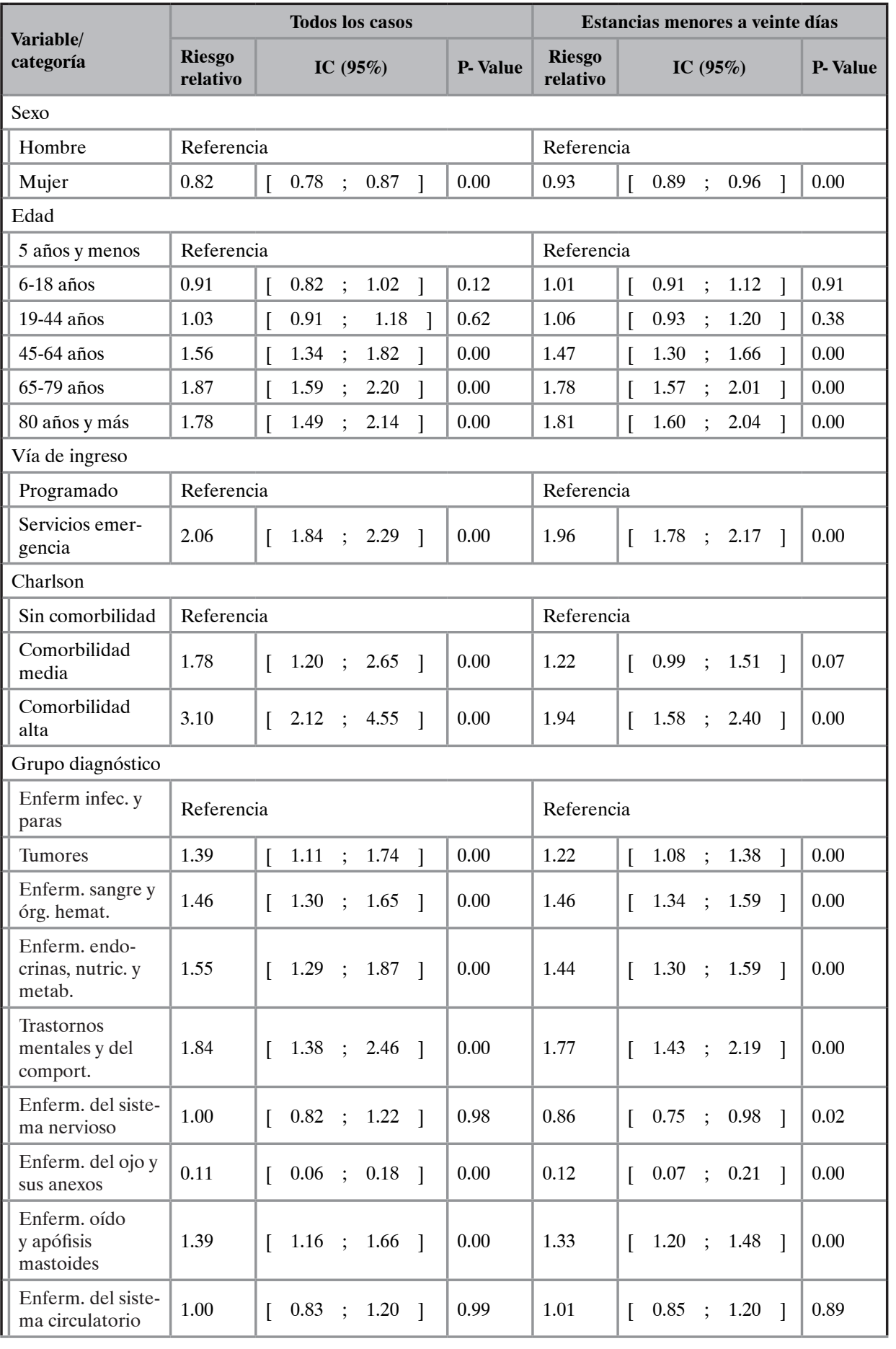




\begin{tabular}{|c|c|c|c|c|c|c|c|c|c|}
\hline \multirow{3}{*}{$\begin{array}{l}\text { Variable/ } \\
\text { categoría }\end{array}$} & \multicolumn{4}{|c|}{ Todos los casos } & \multicolumn{5}{|c|}{ Estancias menores a veinte días } \\
\hline & \multirow{2}{*}{$\begin{array}{l}\text { Riesgo } \\
\text { relativo }\end{array}$} & \multicolumn{2}{|c|}{ IC $(95 \%)$} & \multirow{2}{*}{$\begin{array}{l}\text { P- Value } \\
0.26\end{array}$} & \multirow{2}{*}{$\begin{array}{l}\text { Riesgo } \\
\text { relativo } \\
1.03\end{array}$} & \multicolumn{3}{|c|}{ IC $(95 \%)$} & \multirow{2}{*}{$\begin{array}{l}\text { P- Value } \\
0.69\end{array}$} \\
\hline & & {$\left[\begin{array}{ll}0.75 \\
\end{array}\right.$} & ; 1.08 ] & & & {$\left[\begin{array}{ll}0.90 \\
\end{array}\right.$} & ; 1.18 & & \\
\hline $\begin{array}{l}\text { Enferm. del siste- } \\
\text { ma digestivo }\end{array}$ & 0.80 & {$\left[\begin{array}{ll}0.67 \\
\end{array}\right.$} & $; 0.96]$ & 0.02 & 0.85 & [ 0.75 & ; 0.96 & & 0.01 \\
\hline $\begin{array}{l}\text { Enferm. piel y } \\
\text { tejido subcutáneo }\end{array}$ & 1.31 & {$\left[\begin{array}{ll}0.87 \\
0\end{array}\right.$} & ; 1.96$]$ & 0.19 & 1.06 & [ 0.81 & ; 1.39 & & 0.66 \\
\hline $\begin{array}{l}\text { Enferm. sist. } \\
\text { osteomuscular y } \\
\text { tejido conj. }\end{array}$ & 1.09 & [ 0.88 & ; 1.34$]$ & 0.44 & 1.07 & [ 0.93 & $; \quad 1.23$ & ] & 0.32 \\
\hline $\begin{array}{l}\text { Enferm. del } \\
\text { sistema genitou- } \\
\text { rinario }\end{array}$ & 0.62 & {$[0.50$} & $; 0.78]$ & 0.00 & 0.73 & [ 0.64 & $; \quad 0.83$ & ] & 0.00 \\
\hline $\begin{array}{l}\text { Embarazo, parto } \\
\text { y puerperio }\end{array}$ & 0.57 & {$\left[\begin{array}{ll}{[} & 0.47\end{array}\right.$} & $; 0.68]$ & 0.00 & 0.62 & [ 0.56 & $; \quad 0.69$ & ] & 0.00 \\
\hline $\begin{array}{l}\text { Afecciones perio- } \\
\text { do perinatal }\end{array}$ & 0.57 & {$\left[\begin{array}{ll}0.48 \\
0\end{array}\right.$} & $; 0.67]$ & 0.00 & 0.63 & [ 0.55 & $; \quad 0.71$ & ] & 0.00 \\
\hline $\begin{array}{l}\text { Malformaciones } \\
\text { congénitas }\end{array}$ & 0.99 & {$\left[\begin{array}{ll}{[} & 0.87\end{array}\right.$} & ; 1.13 ] & 0.90 & 1.00 & {$[0.91$} & ; 1.09 & ] & 0.96 \\
\hline $\begin{array}{l}\text { Síntomas, signos } \\
\text { anormales clí- } \\
\text { nicos }\end{array}$ & 1.00 & {$\left[\begin{array}{ll}0.79 \\
0\end{array}\right.$} & ; 1.28$]$ & 0.98 & 1.01 & {$\left[\begin{array}{ll}0.84 \\
\end{array}\right.$} & ; 1.22 & ] & 0.91 \\
\hline $\begin{array}{l}\text { Traumatismos, } \\
\text { envenenamientos } \\
\text { y otros }\end{array}$ & 0.98 & {$\left[\begin{array}{ll}0.81 \\
0\end{array}\right.$} & ; 1.18$]$ & 0.82 & 0.93 & [ 0.81 & ; 1.06 & ] & 0.26 \\
\hline $\begin{array}{l}\text { Factores influyen } \\
\text { estado de salud }\end{array}$ & 0.67 & {$[0.53$} & $; 0.85]$ & 0.00 & 0.64 & {$[0.52$} & $; \quad 0.78$ & ] & 0.00 \\
\hline Otros & 0.14 & [ 0.04 & ; 0.45 ] & 0.00 & 0.16 & [ 0.05 & ; 0.53 & ] & 0.00 \\
\hline \multicolumn{10}{|l|}{ Estado civil } \\
\hline Sin pareja & \multicolumn{4}{|c|}{ Referencia } & \multicolumn{5}{|c|}{ Referencia } \\
\hline Con pareja & 0.88 & {$[0.87$} & $; 0.90 \quad]$ & 0.00 & 0.94 & [ 0.92 & ; 0.95 & ] & 0.00 \\
\hline \multicolumn{10}{|l|}{ Condición de salida } \\
\hline Vivo & \multicolumn{4}{|c|}{ Referencia } & \multicolumn{5}{|c|}{ Referencia } \\
\hline Muerto & 1.37 & [ 1.29 & ; 1.46$]$ & 0.00 & 0.90 & [ 0.85 & ; 0.94 & ] & 0.00 \\
\hline
\end{tabular}

Fuente: elaboración propia con base en datos del Área de Estadística en Salud, Caja Costarricense de Seguro Social

de sistema nervioso central, que resulta estadísticamente significativo.

En la tabla 2 se muestran los resultados del IAE y los intervalos de confianza al $99 \%$ del conjunto de hospitales generales de la CCSS. Se observa que el $43 \%$ de los hospitales registra un IAE superior a $100 \%$, con lo cual se puede asegurar que en ellos existe un inadecuado manejo de las estancias de sus pacientes. Dentro de estos centros hay dos de referencia nacional, tres regionales y cinco periféricos.

Además, se observa que existen diferencias significativas en la adecuación de las estancias entre hospitales de un mismo nivel de 


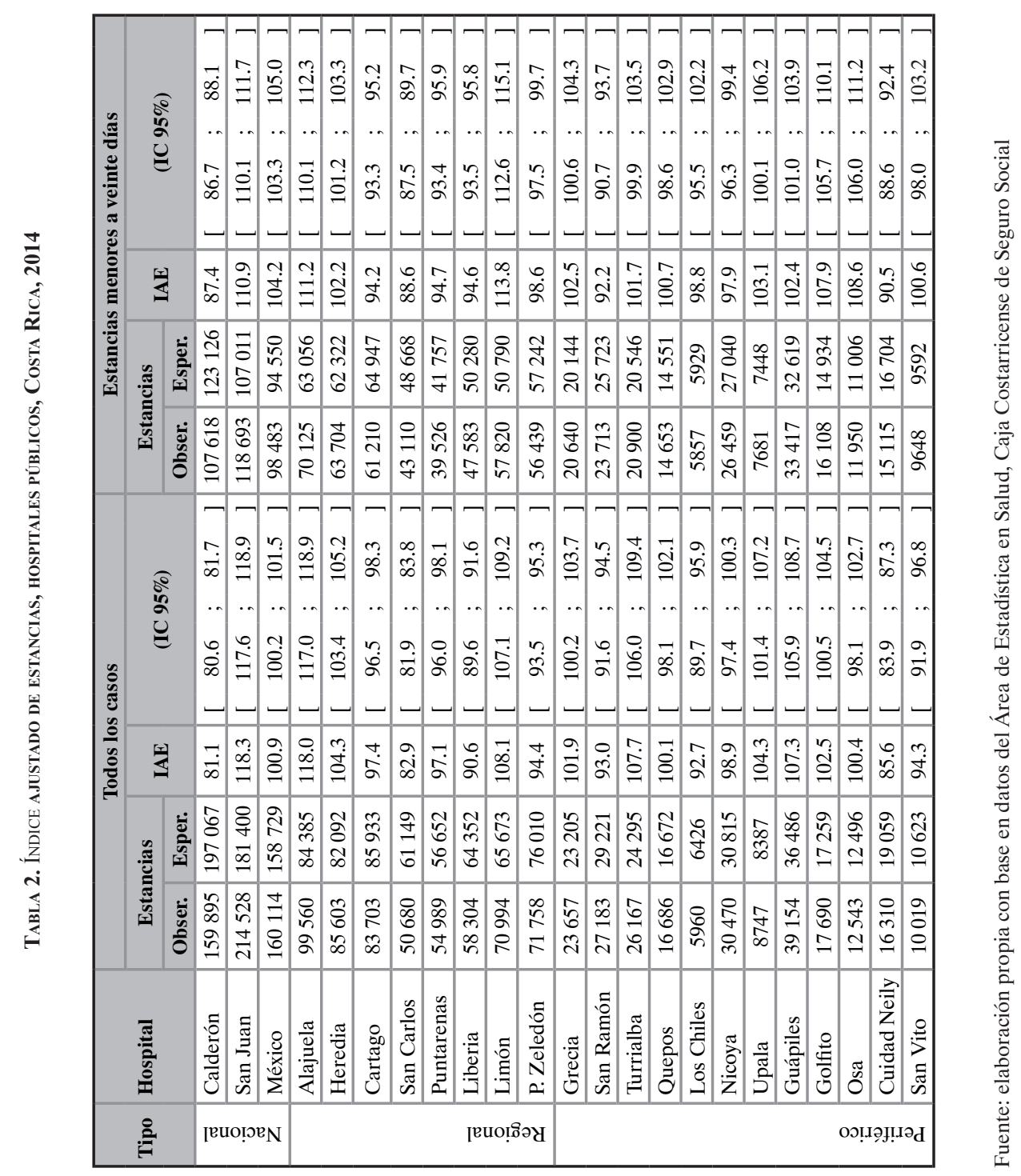


complejidad. Esta variabilidad es similar en los hospitales nacionales y regionales.

En este diagrama se representan las estancias observadas con respecto a una medida de su precisión (defunciones observadas con respecto a las esperadas), de modo que los límites de control forman un "embudo" en torno al resultado objetivo. Para los propósitos de este estudio, el grado de confianza exigido a la estimación ha sido de un 95\% (dos desviaciones estándar, 2 SD).

Todos los datos

Estancias menores a veinte días
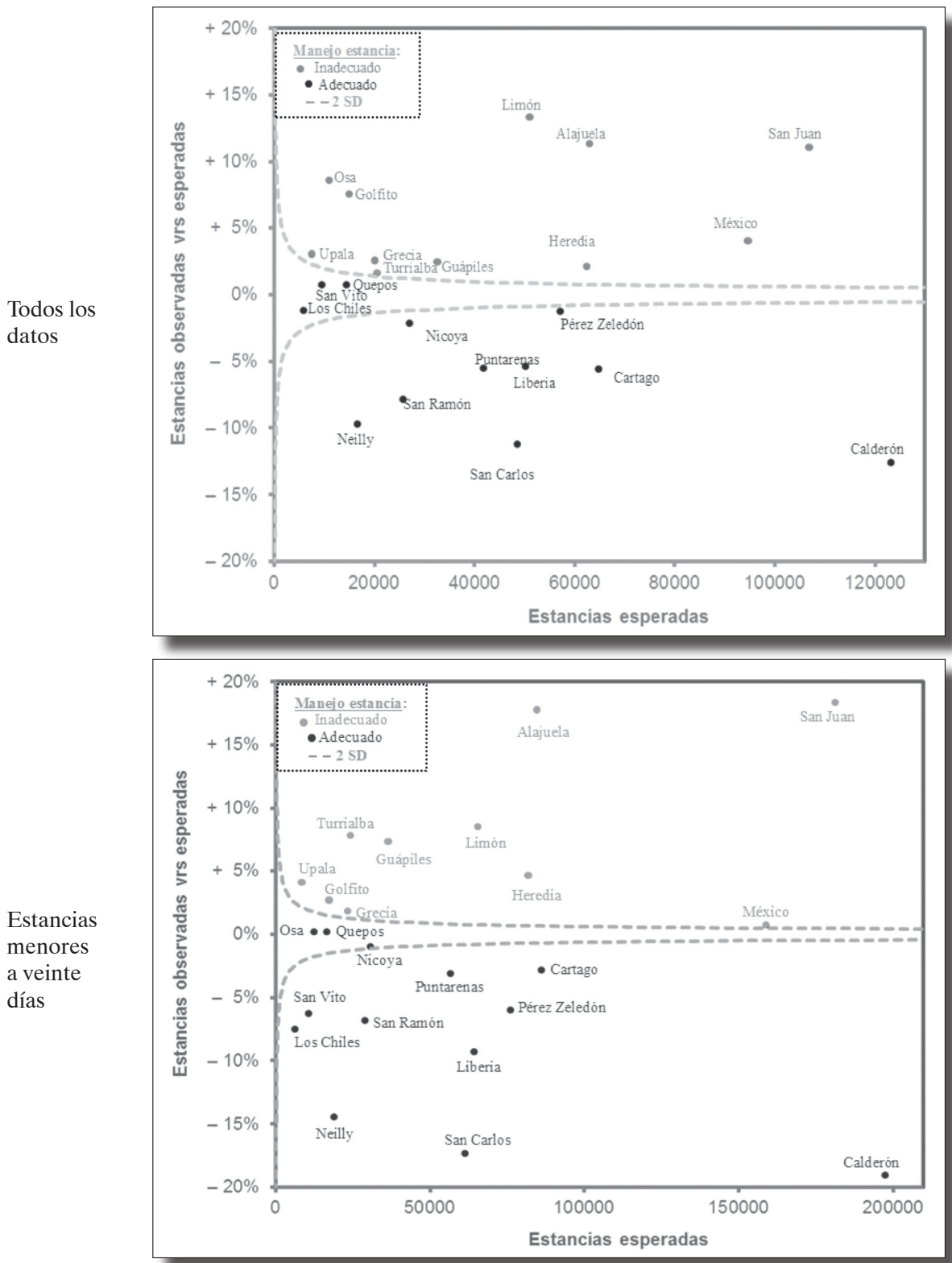

Figura 3. Gráfico de embudo estancias observadas con respecto a las esperadas. Hospitales públicos, COSTA RicA, 2014

Fuente: elaboración propia con base en datos del Área de Estadística en Salud, Caja Costarricense de Seguro Social 
Los resultados del modelo aplicado a la base sin casos extremos no variaron los resultados originales, excepto para los hospitales Turrialba y Osa.

Los puntos que están por encima del límite superior del intervalo de confianza (2 SD) representan hospitales con más estancias que las esperadas, lo que implica que tienen una inadecuada gestión de las estancias.

En la figura 4 se observa que el $43 \%$ de los hospitales registra una gestión inadecuada de las estancias.

\section{Discusión}

Los resultados del modelo de regresión evidencian una asociación estadísticamente significativa entre la estancia registrada y las variables explicativas incluidas. Los hombres presentan una mayor estadía que las mujeres $\mathrm{y}$, asimismo, existe una mayor probabilidad de estancias altas en los grupos de edad avanzada con respecto a los primeros años de vida. Además, el estar casado o en unión libre es una condición que implica una menor estancia.

Se registró una muy alta probabilidad de permanecer más en el hospital si el paciente ingresa por emergencia con respecto a si lo hace de forma programada, en tanto que la existencia de comorbilidad es un factor que influye positivamente en la estadía en el hospital.

Por su parte, los pacientes que fallecen en el hospital tienen una mayor posibilidad de tener una estancia mayor que en cualquier otra condición de salida.

Un estudio para Irán (15) muestra que las estancias inadecuadas se relacionan inversamente con la edad y la comorbilidad. Se registraron mayores estancias inadecuadas en hombres que en mujeres y un menor riesgo de estancias inadecuadas en pacientes que estaban casados. Además, se registra un alto riesgo de estancias inadecuadas en pacientes que ingresan por urgencias. Las causas más frecuentes de días injustificables fueron la espera de los procedimientos de diagnóstico o terapéuticos $(35.1 \%)$ y el retardo de $20.6 \%$ en alta de los pacientes por los médicos, debido a la política conservadora.

En Portugal un estudio encontró que la edad, el tipo de ingreso y el tipo de hospital se asociaron significativamente con altas estancias (8).

Para un hospital de Polonia se encontró una asociación estadísticamente significativa entre las estancias altas y la edad y el tipo de admisión (16).

A partir de la estimación del IAE se determinó que en el $43 \%$ de los hospitales hay una inadecuada gestión de las estancias, dado que registra mayores días de hospitalización que los esperados, de acuerdo con lo que le correspondía por la casuística que atiende. Los resultados del modelo aplicado a la base sin casos extremos no variaron los resultados originales, excepto para los hospitales Turrialba y Osa. El hospital de Turrialba presenta una inadecuada gestión de las estancias cuando se analizan todos los casos, pero cuando se excluyen los casos extremos no existe suficiente evidencia para afirmar que presenta estancias inadecuadas. Caso contrario ocurrió con el hospital de Osa.

En los hospitales con inadecuada gestión de estancias se recomienda elaborar un perfil de eficiencia para identificar en qué categoría diagnóstica mayor el hospital es más eficiente y en cuál menos (17). A partir de estos resultados se podrían desarrollar protocolos, con el fin de estandarizar la prestación de servicios en los diagnósticos con mayor variabilidad en la estancia media. 
Los hospitales que registran estancias superiores a las esperadas deberían realizar un plan de intervención. De la revisión de la literatura (1) se concluye que el factor que más frecuentemente prolonga la estancia en los hospitales es la demora en la realización de procedimientos quirúrgicos y diagnósticos, seguido de la necesidad de atención en otro nivel de complejidad (que evidencia la falta de consolidación de una red de atención), situación sociofamiliar (falta de apoyo a los cuidados en su domicilio, el rechazo de la familia a aceptar el traslado a un hogar de ancianos o las condiciones inadecuadas de las viviendas) y la edad del paciente.

La información obtenida en esta investigación puede servir de insumo para el análisis de la gestión de las camas y las estancias hospitalarias. Un modelo útil y de fácil interpretación para resumir la información de los indicadores de gestión de las camas y las estancias es el diagrama de Pabón-Lasso (18-19). En este se compara la productividad de la cama —-medida como los egresos hospitalarios con respecto a las camas disponibles—, el índice de ocupación — que mide la eficiencia en el manejo de las camas-y el índice de adecuación de las estancias que mide la eficiencia en el manejo de las estancias.

Esta información se puede presentar también a nivel de servicio hospitalario mediante un gráfico de dispersión, con el fin de comparar la ocupación de la cama y la adecuación de las estancias (IAE) (20). Los resultados obtenidos permiten medir la adecuada distribución de las camas entre servicios (se considera un índice de ocupación óptimo si es mayor o igual al 85\%) y determinar si el hospital está manejando adecuadamente las estancias, de tal manera que se permita una utilización óptima de las camas.

\section{Conclusión}

A partir de la información contenida en la base de datos clínico-administrativa del Seguro Público de Salud de Costa Rica, se pudo estimar una estancia ajustada por condiciones del paciente que permitió identificar los hospitales que presentan una gestión inadecuada de las estancias.

Variables sociodemográficas del paciente como la edad y el sexo y condiciones clínicas como el tipo de enfermedad, comorbilidad asociada y urgencia de la atención fueron factores determinantes en la duración de la estadía en el centro hospitalario.

El índice de adecuación de las estancias puede sustituir al indicador de estancia media cruda como insumo para el análisis de la gestión de las camas y las estancias hospitalarias.

\section{Referencias bibliográficas}

1. Ceballos-Acevedo T, Velásquez-Restrepo PA, JaénPosada JS. Duración de la estancia hospitalaria. Metodologías para su intervención. Rev Gerenc Polít Salud [Internet]. 2014; 13(27):274-95. Disponible en: http://dx.doi. org/10.11144/Javeriana. rgyps13-27.dehm

2. Tamargo T, Jiménez R. El ajuste por riesgo en la evaluación del desempeño hospitalario. Rev Cubana Salud Pública. 2009; 35.

3. Martín J, López MP. La medida de la eficiencia en las organizaciones sanitarias. Presupuesto y Gasto Público. 2007; 49:139-61.

4. Copnell B, Hagger V, Wilson SG, Evans SM, Sprivulis PC, Cameron PA. Measuring the quality of hospital care: an inventory of indicators. Intern Med J. 2009; 39(6):352-60.

5. Brownell M, Roos N. Variation in length of stay as a measure of efficiency in Manitoba hospitals. Can Med Assoc J. 1995; 152:675-82.

6. Jiménez R. Indicadores de calidad y eficiencia de los servicios hospitalarios. Una mirada actual. Revista Cubana Salud Pública. 2004; 30(1).

7. González Angulo I. Relación entre el prestador de servicio de salud y la estancia prolongada en el hospital. Revista Conamed. 2009; 14(4).

8. Freitas A, Silva-Costa T, Lopes F, Garcia-Lema I, Teixeira-Pinto A, Brazdil P, Costa-Pereira A. Factors influencing hospital high length of stay outliers. BMC Health Services Research. 2012; 12(1). 
9. Casas M. Los grupos relacionados con el diagnóstico. Experiencia y perspectivas de utilización. Madrid: Masson; 1991.

10. Rivero A, Sendino M, Cózar R, Pascual M, García J, García C, et al. Análisis y desarrollo de los GDR en el Sistema Nacional de Salud [Internet]. Madrid: Ministerio de Sanidad y Consumo; 1999 [acceso: 19 de mayo del 2014]. Disponible en: http://www. msps.es/estadEstudios/estadisticas/docs/analisis.pdf

11. Moya L. Aplicación de los grupos de diagnósticos relacionados a la gestión del sistema nacional de servicios de hospitalización de la CCSS. Rev Cienc Adm Financ Segur Soc. 1998; 6:13-33.

12. Quan H, Li B, Couris C, Fushimi K, et al. Updating and validating the Charlson Comorbidity Index and Score for Risk Adjustment in hospital discharge abstracts using data from 6 countries. American Journal of Epidemiology. 2011; 173(6):676-82.

13. Newcombe RG. Two-sided confidence intervals for the single proportion: Comparison of seven methods. Stat Med. 1998; 17:857-72.

14. Organización Mundial de la Salud. Lista corta para el análisis de mortalidad [Internet] [acceso: 3 de marzo del 2016]. Disponible en: http://www.paho. org/spanish/dd/ais/be_v23n4-intro_listas_cortas.htm

15. Ghods A, Khabiri R, Raeisdana N, Ansari M, et al. Predictors of inappropriate hospital stay: Experience from Iran. Global Journal of Health Science. 2015; (3):82-89.

16. Cyganska M. The impact factors on the hospital high length of stay outliers. Procedia Economics and Finance. 2016; 39:251-5.

17. Green L, Nguyen V. Strategies for cutting hospital beds: The impact on patient service. Health Serv Res. 2001; 36:421-41.

18. Pabon-Lasso H. Evaluating hospital performance through simultaneous application of several indicators. Bulletin of the Pan American Health Organization. 1986; 20:341-57.

19. Bahadori M, Sadeghifar J, Hamouzadeh P, et al. Combining multiple indicators to assess hospital performance in Iran using the Pabon Lasso Model. Australasian Medical Journal. 2011; 4:175-9.

20. Morera M. Gestión de estancias y ocupación de camas de hospitales públicos de Costa Rica. Acta Méd Costarric. 2014; 56(3). 